\title{
Aerial Seismology Using Balloon-Based Barometers
}

\author{
Siddharth Krishnamoorthy ${ }^{(0)}$, Voon Hui Lai, Attila Komjathy, Michael T. Pauken, James A. Cutts, \\ Raphaël F. Garcia, David Mimoun, Jennifer M. Jackson, Daniel C. Bowman, \\ Ervan Kassarian, Léo Martire, Anthony Sournac, and Alexandre Cadu
}

\begin{abstract}
Seismology on Venus has long eluded planetary scientists due to extreme temperature and pressure conditions on its surface, which most electronics cannot withstand for mission durations required for ground-based seismic studies. We show that infrasonic (low-frequency) pressure fluctuations, generated as a result of ground motion, produced by an artificial seismic source known as a seismic hammer, and recorded using sensitive microbarometers deployed on a tethered balloon, are able to replicate the frequency content of ground motion. We also show that weak, artificial seismic activity thus produced may be geolocated by using multiple airborne barometers. The success of this technique paves the way for balloon-based aeroseismology, leading to a potentially revolutionary method to perform seismic studies from a remote airborne station on the earth and solar system objects with substantial atmospheres such as Venus and Titan.
\end{abstract}

Index Terms-Balloons, infrasound, remote sensing, seismology, titan, venus.

\section{INTRODUCTION}

$\mathbf{V}$ ENUS, often called earth's planetary twin due to its similar mass and size [1], has remained an enigma for planetary scientists despite visits by multiple orbiters and probes over the last 6 decades. Since the first visit by Mariner 2

Manuscript received February 6, 2019; revised July 8, 2019; accepted July 24, 2019. Date of publication August 21, 2019; date of current version November 25, 2019. This work was supported by the Sandia National Laboratories is a multimission laboratory managed and operated by National Technology and Engineering Solutions of Sandia, LLC, a wholly owned subsidiary of Honeywell International, Inc., for the U.S. Department of Energy's National Nuclear Security Administration, under Contract DE-NA0003525. The works of S. Krishnamoorthy, A. Komjathy, M. T. Pauken, and J. A. Cutts were supported by the Jet Propulsion Laboratory, California Institute of Technology, under a contract with the National Aeronautics and Space Administration. The works of V. H. Lai and J. M. Jackson were supported by the W. M. Keck Institute for Space Studies. The works of R. F. Garcia, D. Mimoun, E. Kassarian, L. Martire, A. Sournac, and A. Cadu were supported in part by ISAE-SUPAERO, in part by the Fondation ISAE-SUPAERO, in part by the la Délégation Générale de l'Armement (DGA), in part by the le Région Occitanie, and in part by the le Centre National d'Études Spatiales (CNES). (Corresponding author: Siddharth Krishnamoorthy.)

S. Krishnamoorthy, A. Komjathy, M. T. Pauken, and J. A. Cutts are with the NASA Jet Propulsion Laboratory, California Institute of Technology, Pasadena, CA 91109 USA (e-mail: siddharth.krishnamoorthy@jpl.nasa.gov).

V. H. Lai and J. M. Jackson are with the Seismological Laboratory, California Institute of Technology, Pasadena, CA 91125 USA.

R. F. Garcia, D. Mimoun, E. Kassarian, L. Martire, A. Sournac, and A. Cadu are with the Institut Supérieur de l'Aéronautique et de l'Espace-SUPAERO, 31400 Toulouse, France.

D. C. Bowman is with the Sandia National Laboratories, Albuquerque, NM 87185 USA.

This article has supplementary downloadable material available at http://ieeexplore.ieee.org, provided by the author.

Color versions of one or more of the figures in this article are available online at http://ieeexplore.ieee.org.

Digital Object Identifier 10.1109/TGRS.2019.2931831 in 1962, several probes and orbiters have confirmed that, while it appears similar to earth, Venus diverged from the earth during the planetary evolution process. Venus' atmosphere contains $96.5 \%$ carbon dioxide, which causes surface temperatures of greater than $460{ }^{\circ} \mathrm{C}$; the surface atmospheric pressure is greater than $90 \mathrm{~atm}$-an atmosphere so thick that it alters the length of the day and the rotation of the planet by exerting a torque on the solid planet [3].

The earth's interior has been rigorously investigated and the interaction of the solid planet and the atmosphere has been studied in great detail. Much of the information about the earth's interior has come from performing seismic studies using ground stations that detect and characterize seismic waves generated as a result of tectonic and volcanic activity [4]-[7]. NASA's InSight mission, which landed on Mars in November 2018, seeks to investigate the red planet's interior [8]. On Venus, however, the possibility of a long-duration ground station is remote, as the adverse surface conditions cause rapid failure of electronic systems and instruments. Even relatively simple but hardened oscillator circuits have lasted only up to $161 \mathrm{~h}$ when subject to Venus surface conditions in the laboratory [9] and materials used to manufacture electronics have been tested for up to 42 days, exhibiting varying degrees of degradation [10].

It has previously been hypothesized that ground motion on Venus could be detected and characterized using infrasonic waves (or infrasound, pressure waves with a frequency less than $20 \mathrm{~Hz}$ ) generated by quakes and volcanic activity through coupling between the solid planet and the atmosphere [11]. Infrasound is known to travel large distances from the originating event and could be characterized using barometers suspended from balloons at approximately $60-\mathrm{km}$ altitude on Venus, where the temperature and pressure are more earthlike ( $0{ }^{\circ} \mathrm{C}$ and 1 atm) [12], [13], and much longer mission lifetimes compared to surface missions can be achieved. Furthermore, seismoacoustic coupling on Venus is known to be up to 60 times more efficient than on the earth [14]. Therefore, if the seismic activity can be detected and characterized using infrasound on earth, the same can be done for Venus. Although the detection limit for seismic activity on Venus using this technique depends on a large number of parameters such as balloon altitude, signal propagation path, and wind speed, our preliminary calculations with 1-D geometric attenuation indicate that quakes with surface magnitudes as low as 3.0 may be detectable at $60-\mathrm{km}$ altitude and over $100 \mathrm{~km}$ away from the quake epicenter, if pressure fluctuations on the order 
of $10^{-3}$ Pa can be measured. A more detailed analysis of detection limits is currently pending.

Balloon-based infrasound studies on earth were pioneered by the U.S. Air Force as part of Project Mogul in the late 1940s for monitoring nuclear explosions conducted by the Soviet Union. Coffman [15] and Wescott [16] pioneered early scientific work in airborne infrasound-while Coffman designed a microphone that was compatible with balloon flights, Wescott deployed these microphones on high-altitude balloons to study atmospheric turbulence. Wescott [16] used two barometers on a tether, a setup that we have also adopted in this work, to discriminate upward-propagating acoustic signals to distinguish atmospheric turbulence from hydrodynamic noise of the wind flowing past the microphone. Following this work, there was a nearly 50-year gap in balloon-based infrasound studies, until Bowman and Lees [17], [18] flew infrasound payloads as part of NASA's High-Altitude Student Platform (HASP) program and demonstrated much lower levels of background wind noise in the earth's stratosphere compared to groundbased infrasound sensors. A more detailed background of the evolution of balloon-based infrasound studies can be found in [19].

Concurrently with the HASP flights, we initiated a program in 2016 to demonstrate the ability of balloon-borne barometers to detect and characterize seismic activity from a floating platform using artificial and, eventually, natural seismic sources. In the current work, a "seismic hammer" was used as a source to produce weak, artificial seismic signals, which generated acoustic signals with amplitudes of the order of $0.1 \mathrm{~Pa}$ at a slant range of approximately $300 \mathrm{~m}$. These seismoacoustic signals were detected using balloon-borne barometers [20]. We demonstrate, here, that balloon-borne barometers can do much more than just detect seismoacoustic signals- they are able to effectively characterize the frequency content of the ground motion and geolocate seismic sources without ever touching the ground. We will first briefly describe the seismic hammer experiment, and a discussion of the ground motion profile will follow. We will then demonstrate important aspects of performing seismology with airborne barometers using one and two barometers-detection, characterization, and geolocation. A demonstration showing the geolocation and spectral characterization of seismic signals using acoustic signals measured on a balloon platform has never been performed before and serves as a proof of concept that will pave the way for a new approach to seismology, which can assist in studying the interiors of planets without needing to land on them.

\section{SEISMIC HAMMER EXPERIMENT}

The seismic hammer experiment was performed in Pahrump, Nevada on June 28, 2017. The hammer, which uses a weight drop-catch-based source, repeatedly dropped a 13-t mass to generate artificial seismic signals [21]. The seismic hammer produces weak but highly repeatable seismic signals by releasing approximately 0.19 MJ of energy on the surface, compared to approximately 4 GJ released by the explosion of 1 ton of trinitrotoluene (TNT) and tens of gigajoules for moment magnitude 3-4 earthquakes [22].
By virtue of its vertically dropping mass, the seismic hammer produces predominantly vertical ground motion.

A total of 108 shots occurred over a duration of more than $4 \mathrm{~h}$ between 6:53 am and 11:30 am local time. Vertical ground motion from the shots was monitored using a large array of Sunfull geophones with a $2-\mathrm{Hz}$ lower corner frequency. The geophones were buried $10-15 \mathrm{~cm}$ below the surface to reduce noise and placed in a dense network surrounding the hammer, with line profiles radiating away from it between 100 and $2000 \mathrm{~m}$ away. Ground motion data were recorded using the iSeis Sigma hardware, which operates at 2000 samples per second and uses timing information from the Global Positioning System (GPS) as a clock. The layout of the geophone network is included in Fig. 1. Paroscientific Digiquartz 6000-15B-IS barometers were deployed to capture the acoustic signal generated by ground motion. We present analysis from two barometers suspended using a tether from a moored balloon (aerostat) anchored 100-200 $\mathrm{m}$ away. The aerostat was active during shots 70-108 of the sequence. Detailed information about the experimental setup can be found in [20]. The seismic hammer experiment was the first experiment to successfully detect infrasound from ground motion on a balloon platform.

\section{Ground Motion Characteristics}

We utilized the dense geophone network surrounding the hammer to study the properties of ground motion near the hammer impact site. The ground motion signal was found to be saturated in several of the geophones close to the hammer due to insufficient dynamic range. Although most of the geophones within a $12-\mathrm{m}$ radius of the hammer were rendered unusable, we were able to recover usable data from many of the saturated geophones using the Projection Onto Convex Sets (POCSs) technique [23], [24]. Ground motion traces from multiple shots are shown in Fig. 2 of the supplementary material. The shots are highly repeatable, demonstrated by the high similarity in the seismic waveforms, implying that the hammer may be treated as a constant source. The recovered geophone signals offer a glimpse into the behavior of the ground motion near the hammer-the spectral content of ground motion was, as expected, found to vary greatly as a function of distance from the hammer, as the signals propagated through the dispersive ground. In addition, spectrograms generated using the continuous wavelet transform (CWT) with Morlet wavelets [25], [26] show that ground motion is also affected strongly by the azimuth relative to the hammer, as shown in Fig. 1. Variation in ground motion across the azimuth reflects the heterogeneity of the subsurface geology within the basin bounded by two northwest and southwest trending ridges (see Fig. 1 of the supplementary material). The lack of isotropy in ground motion proved to be a useful diagnostic tool for the barometer signal - the aerostat floated in different directions relative to the hammer at different times, and the azimuthal variation in the ground motion was found to be imprinted in the acoustic signal generated by ground motion as well. This is discussed in more detail in Section IV.

The acoustic regime may be classified as "far field" if the distance between the source and detector is larger than the 

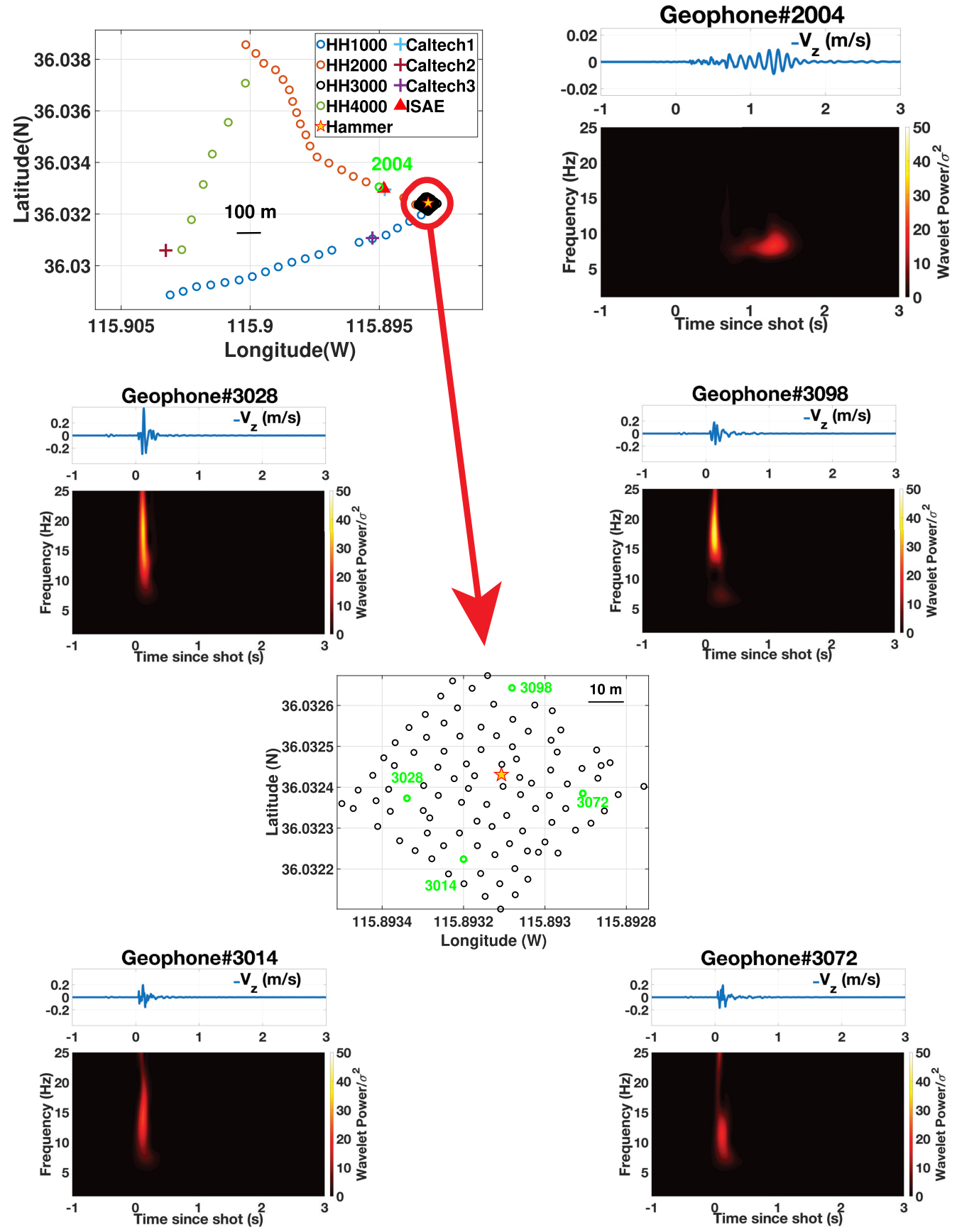

Fig. 1. Analysis of ground motion near the seismic hammer. (Top left) Map of all seismometers deployed near the hammer. The "HH1000-4000" seismometers are single-axis Sunfull geophones. The "Caltech" and "ISAE" seismometers are three-axis Trillium seismometers (not utilized for results presented here). The HH3000 series was deployed in a dense network around the hammer, shown by black circles. The ground motion time series and CWT spectrogram show the variation of ground motion based on direction and distance from the hammer. Geophones 3098, 3072, 3014, and 3028 are at approximately $24 \mathrm{~m}$ from the hammer but in different directions. Subsurface heterogeneous structures can cause variations in ground motion. As the seismic signal propagates out to the location of geophone 2004, the signal decreases in strength and retains only its lower frequency components.

Rayleigh distance $R$, given by [27], [28]

$$
R=\frac{S}{\lambda}
$$

where $S$ is the surface area of the source and $\lambda$ is the wavelength of the acoustic wave under consideration. The highest frequency modes in ground motion are detected close to the hammer, which decay rapidly with distance. To estimate the largest Rayleigh distance, we consider a surface of radius $25 \mathrm{~m}$ centered at the hammer site as a "baffle" that generates the acoustic waves detected by the barometers (similar to the treatment of Jones et al. [29]). This is a conservative estimate that encompasses almost the entirety of the geophone network close to the hammer. To obtain the Rayleigh distance for the shortest wavelength, we use $20 \mathrm{~Hz}$ as the highest frequency detected by the barometer and use $303 \mathrm{~K}$, the lowest 


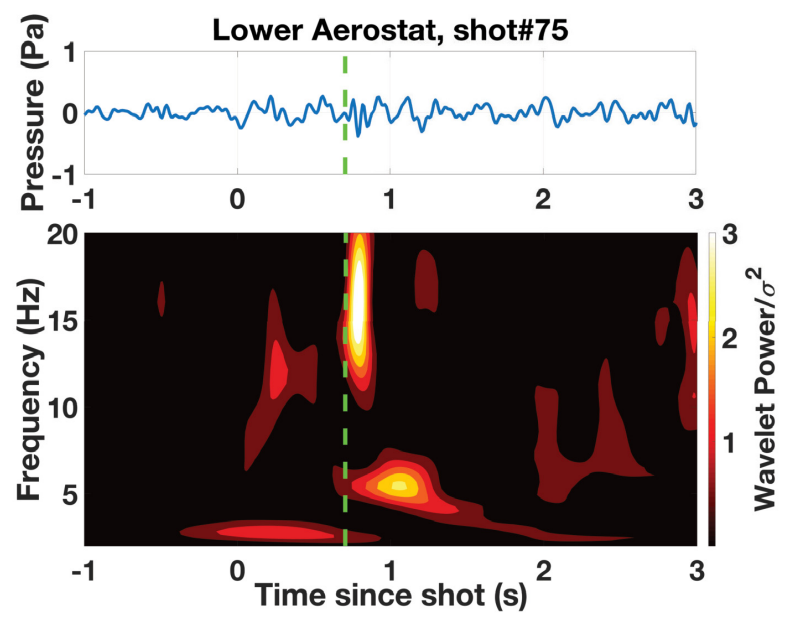

(a)

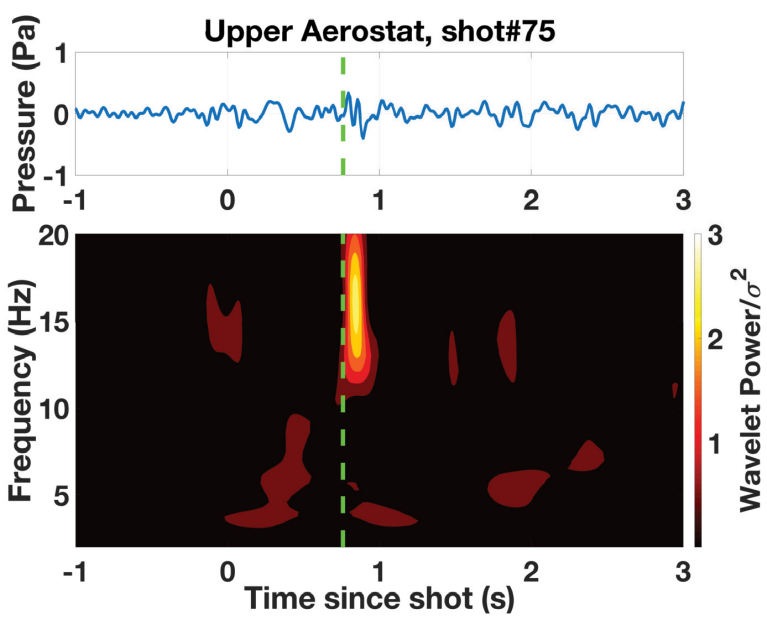

(b)

Fig. 2. Representative signals from shot 75 as detected by the (a) lower and (b) upper aerostat barometers. The signal has poor SNR in the time domain due to wind noise contamination and balloon wake effects but appears clearly in the CWT-produced spectrogram. Green dashed line: expected TOA after the shot, based on the barometer's distance from the hammer site. The signal arrives a short time later at the upper aerostat. The delayed arrival at lower frequencies in the lower aerostat is likely the surface wave passing underneath the barometer. Other shots are shared in movies S1 and S2 of the supplementary material.

temperature recorded during the experiment to calculate the speed of sound $(348.95 \mathrm{~m} / \mathrm{s})$. Under these assumptions, the minimum wavelength is $17.44 \mathrm{~m}$ and the maximum possible Rayleigh distance is $112.58 \mathrm{~m}$. The nearest aerostat barometer was always greater than $148 \mathrm{~m}$ away from the hammer site. Thus, the acoustic signals detected in this experiment are classified as "far field" signatures of the artificial seismic event, and in a similar acoustic regime as a balloon attempting to detect a natural earthquake (larger "baffle radius" but much longer wavelengths) in the stratosphere.

\section{Single Barometer Aerial Seismology}

Ground-based infrasound stations often deploy several barometers and channels to probe arriving infrasound signals. Although arrays of barometers are comparatively challenging to deploy in the air, in this section, we show that individual barometer units suspended from balloons can also effectively characterize the frequency content of seismic activity, thereby showing the replication of ground motion to within a multiplicative factor. Pressure signals discussed in Section IV-A have been bandpass filtered between 2 and $20 \mathrm{~Hz}$ to maintain the infrasonic nature of the signal while reducing interference from balloon motion and wind noise, which dominate in the sub-2-Hz band. Pressure signals used in Section IV-B have a narrower filter passband $(10-20 \mathrm{~Hz})$ to further enhance the seismically induced infrasound perturbation compared to the background.

\section{A. Signal Detection}

In our previous work [20], we showed that the pressure signals on the aerostat barometers could be stacked using the signals' expected arrival time as a reference to enhance the SNR. Furthermore, since the individual traces were noisy, empirical wavelet transform (EWT) techniques [30] were used to construct similarity metrics that demonstrated the detection of the seismoacoustic signal from individual shots. We applied CWT to individual shot traces from the upper and lower aerostat barometers and found this technique to produce higher SNR spectrograms than the EWT technique we had previously used-the CWT considers many more scales in the wavelet transform than the EWT, which is based on the discrete wavelet transform, and generates a spectrogram that shows signal arrival more clearly. Fig. 2 shows representative detections of the hammer strike by the upper and lower aerostat barometers-even though the SNR in the timedomain signal is poor (due to the weak signal and wind noise interference), the spectrograms of the signal clearly show regions of increased wavelet power in the same frequency band as the ground motion shown in Fig. 1. The regions of increased intensity arrive precisely at the expected arrival time, denoted by the dotted green line, which also serves to disambiguate the hammer signal from sporadic increases in wavelet power due to wind interference. The hammer produces an audible high-frequency sound when it strikes the ground. This "direct" acoustic wave is filtered out by retaining only acoustic waves between 2 and $20 \mathrm{~Hz}$. Of the 39 shots that each barometer was active for, the lower aerostat shows increased wavelet power concurrent with the expected arrival time of the signal for 34 shots. The upper aerostat, which was affected severely by the wake of the balloon flying above it and shows even poorer SNR in the time domain, is also able to detect 28 shots in the 36 available shot traces using the CWT technique (three-shot traces were rejected in the case of the upper aerostat due to glitches in the data recording software). All time traces and spectrograms from the available shots for both barometers are shared in movies S1 and S2 of the supplementary material accompanying this paper.

\section{B. Frequency Response}

The frequencies at which the wavelet power shows a peak in the shot traces available for both barometers appear 

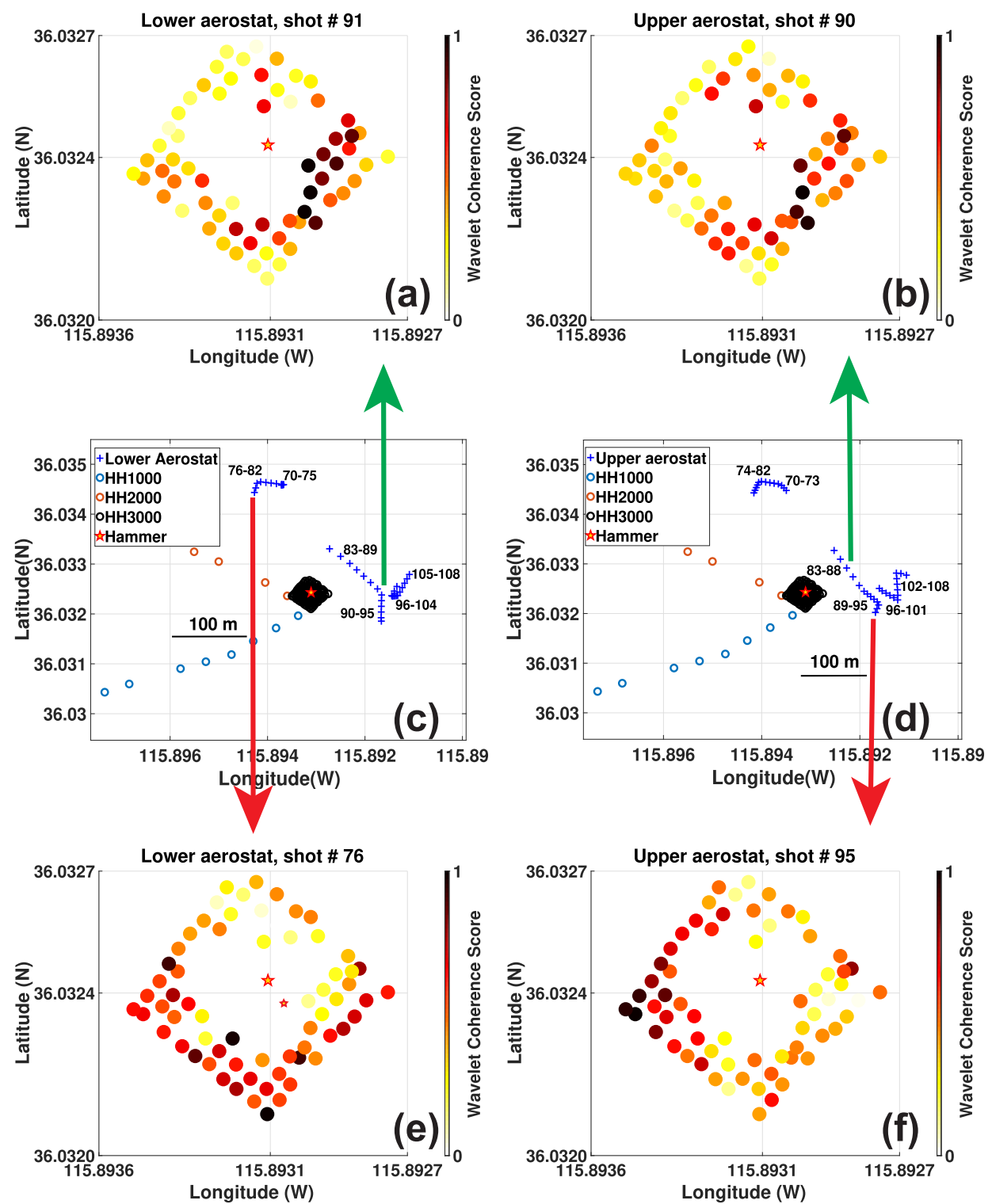

Fig. 3. Wavelet coherence score for both aerostat barometers with available geophone traces from near the hammer. The maps in (c) and (d) show the estimated location of the airborne barometers at the shot time obtained from their GPS location tags (denoted by blue crosses), with the shot number indicated in black text next to the barometer position. The geophone positions are shown by black, orange, and blue circles. The wavelet coherence score indicates spectral similarity between the barometer and ground motion signal as recorded at each geophone in the HH3000 series. Since the ground motion varies with the direction, the coherence score can be used to detect the direction of the barometer with respect to the hammer. In the successful cases [shown in (a) and (b)], the geophones in the direction of the barometer have a higher score than those in the opposite direction. However, wind noise also causes cases where this technique fails to produce an accurate wavelet coherence map [shown in (e) and (f)].

to change with a shot number. This is reminiscent of the change in wavelet power exhibited in the ground motion as a function of the azimuth. During the experiment, the aerostat was anchored at two different locations, and the prevailing wind caused the balloon to drift to different azimuths with respect to the hammer. We investigated the ability of the barometers to replicate the frequency content of ground motion and examined their sensitivity to the azimuthal variation in ground motion by comparing the pressure signal received by the barometers with each of the usable geophones near the hammer. Jones et al. [29] have previously examined the infrasound signal from subsurface chemical explosions by treating the surface above "ground zero," i.e., the location of the explosion, as a baffle that modulates the air above it. They found that the infrasound signature $p(R, t)$ at a range $R$ and time $t$ can be obtained by solving the Rayleigh integral [27] for pressure waves radiating from the baffle $S$ as

$$
p(R, t)=\rho_{0} \int_{S} \frac{a\left(x^{\prime}, y^{\prime}, t-R / c_{0}\right)}{2 \pi R} d S
$$

where $\rho_{0}$ and $c_{0}$ are the ambient air density and the speed of sound, and $a\left(x^{\prime}, y^{\prime}, t\right)$ is the acceleration profile of the baffle. The Rayleigh integral assumes a uniform background fluid and ignores atmospheric variations. This approximation is valid for local propagation and was found to be accurate even up to a slant range of $18.3 \mathrm{~km}$ by Banister and Hereford [31]. 

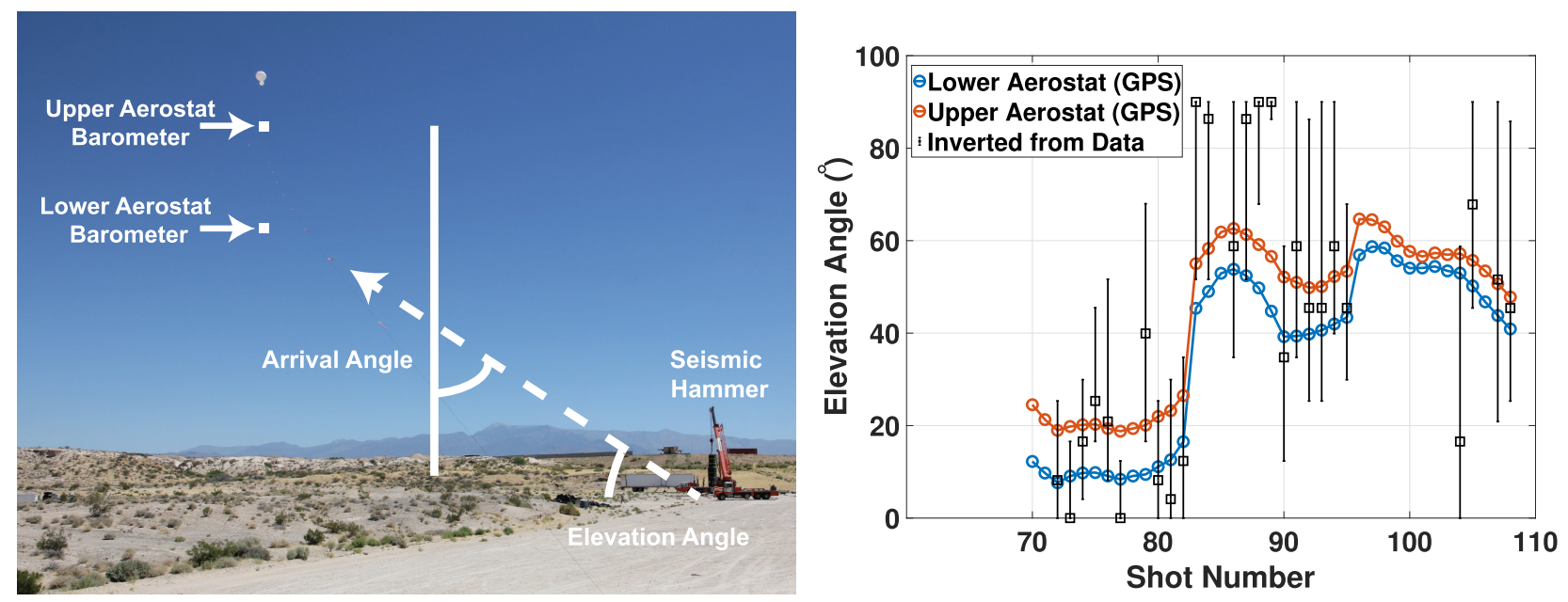

Fig. 4. AOA determined from the time delay between the lower and upper aerostat barometers. The time delay was obtained using CWT spectrogram correlations since arrivals were clearest in the time-frequency domain (method described in Section II-D of the supplementary material). The elevation angles inverted from data show the same trend as those obtained from GPS positioning. The large error bars are a result of broad correlation peaks in the time-frequency domain.

Thus, the resultant infrasound signal at the barometer is a linear combination of the signal produced by the oscillation of different parts of the surface near the seismic hammer, and comparing the spectral similarity of the pressure signature with individual geophones is potentially a many-to-one problem. Banister and Hereford [31] have previously found that pressure signals taken above underground nuclear explosions agree well with the Rayleigh integral computed from ground motion time series. Martire et al. [32] also studied seismoacoustic radiation using numerical simulations and found the evidence of interference in the infrasound signal emerging from different parts of the radiating surface.

The spectral similarity of the signals was compared using the wavelet coherence [26], [33], [34], which was computed for pairings of the barometer signal with each near-field geophone based on signal arrival time. Based on the spectrograms computed using the CWT method, it was determined that the energy of the hammer was predominantly concentrated in the $10-20-\mathrm{Hz}$ band. Therefore, the barometer signals were filtered between 10 and $20 \mathrm{~Hz}$ prior to performing wavelet coherence. The wavelet coherence method is described in detail in Section II-C of the supplementary material. Fig. 3 shows the "wavelet similarity" score based on the wavelet coherence between the ground motion signal at a given geophone and the barometer signal. The similarity score is computed at the time a pressure signal is expected to arrive at the barometer from the geophone's location and is scaled between 0 and 1 . Values close to 1 (darker dots) indicate a high level of spectral similarity between the given geophone's signal and that of the barometer. If the barometer is indeed sensitive to variation in ground motion, then the pressure signal it measures should be more similar to those geophones that are closer to it than the hammer, compared to those that are further away. In the rendering shown in Fig. 3, this would mean that the geophones in the direction of the balloon show darker spots than those facing away from it. Greater coherence is exhibited between the barometer and geophones in the direction of the balloon as compared to the geophones in the direction facing away from the balloon at a total of 22 out of 39 times for the lower aerostat barometer and 21 out of 36 times for the upper aerostat barometer-a high success rate considering the unfavorable SNR in the raw signal. The failures are primarily due to the low SNR in the pressure signal, but also because the infrasound signal in the far-field at any given time is a combination of the infrasound disturbance produced by the motion of different areas around the hammer. Nonetheless, given the success rate of this technique and similarity in spectrogram records between the geophones near the hammer, and balloon-based barometers compel us to conclude that the pressure signal is able to retain much of the infrasonic frequency content of the vertical ground motion. This result indicates that the ground motion waveforms from the seismic activity may be effectively measured without landing on the surface.

\section{Dual Barometer Aerial Seismology}

Since pressure is a scalar, a single pressure measurement is unable to geolocate the source of an infrasound event. However, with two barometers on a tether, the time difference between the arrival of the signal at the two barometers can yield information about the location of the source and the vertical direction of propagation. Geometrical estimation techniques such as time of arrival (TOA), time difference of arrival (TDOA), and angle of arrival (AOA) are frequently used for geolocation in wireless networks and in global navigation satellite systems (GNSS) [35]-[37]. Infrasound arrays deployed by the infrasound monitoring stations (IMS) network of the Comprehensive Nuclear-Test-Ban Treaty Organization (CTBTO) often use arrays spread out over squares of kilometers to determine the azimuth of arrival and signal speed [38]. In our experiment, the two barometers were separated by a 


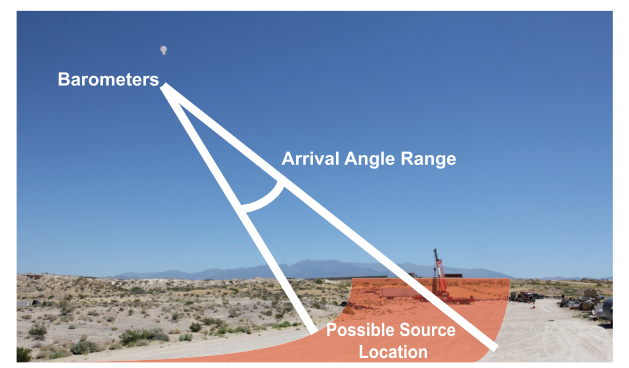

(a)

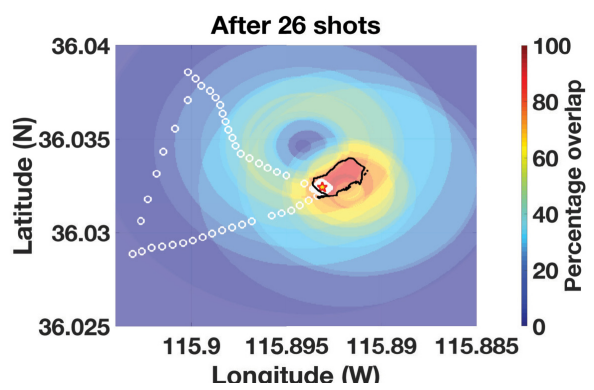

(b)

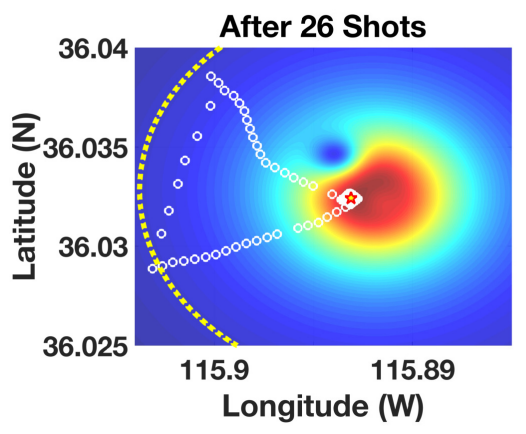

(c)

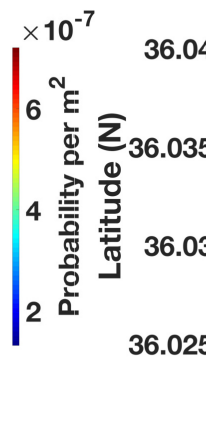

36.025

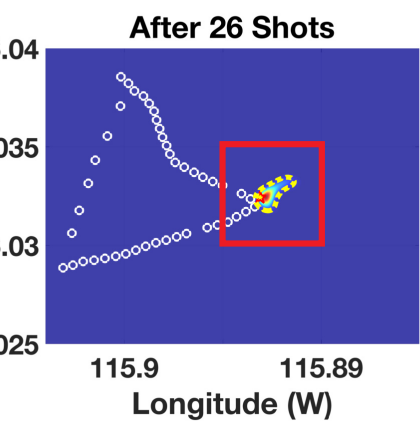

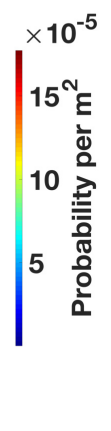

(d)

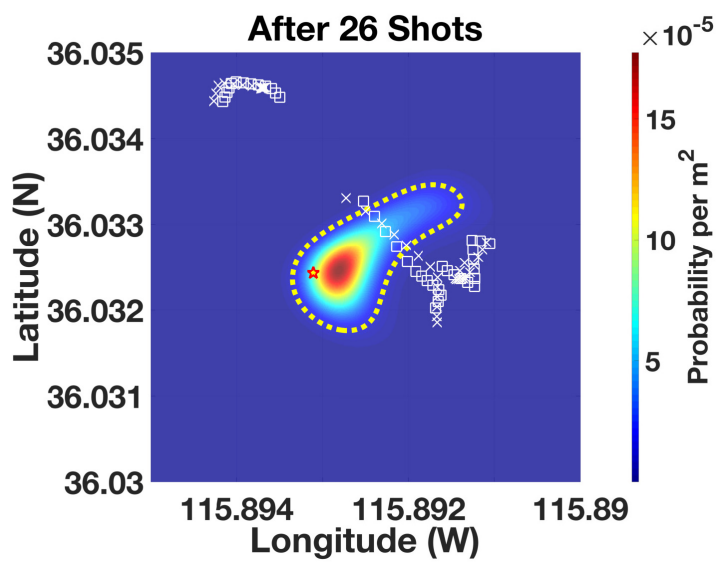

(e)

Fig. 5. Geolocation using the time delay obtained in Fig. 4. (a) Arrival angle range can be projected to the ground to give a possible annulus for the source. (b) After superposing annuli from 26 identical shots, the hammer is within the $80 \%$ overlap region demarcated by the black line. The systematic bias of making measurements only north and east of the hammer is visible. (c) Using a Bayesian estimate to determine the hammer location (assumed unknown a priori in a search area of $4 \mathrm{~km}^{2}$ ), if we consider the error due to interpolating barometer positions once every $100 \mathrm{~s}$, we obtain a semimajor axis of $960 \mathrm{~m}$ (upper bound) for the $90 \%$ likelihood region (demarcated with the yellow dashed line). (d) If we account only for GPS error in barometer positions (lower limit), we recover an ellipse with semimajor axis of approximately $80 \mathrm{~m}$ that contains the source with $90 \%$ likelihood, with the hammer appearing approximately 26-m west-south-west of the peak probability density area due to bias introduced by making measurements only to the north and the east of the hammer. The geophone network is denoted by white circles [HH3000 series hidden in (d) for clarity] and the hammer is located at the star. (e) Zoomed-in view of the inset marked by the red rectangle in (d), white crosses and squares show the locations of the lower and upper aerostat barometers, respectively. The evolution of the overlap pattern from 1 to 26 shots is shown in Movie S6 of the supplementary material.

50-m tether. The low SNR of the raw signal precluded the use of time-domain correlation methods to determine the TDOA for the two barometers. However, we note that the infrasound signature of several shots is very clear for both barometers in the time-frequency domain. Thus, it was possible for us to use this data to generate the TDOA for the pair of aerostat barometers. Fig. 4 shows the AOA estimated from the TDOA technique compared to the angle of elevation determined from GPS positions. Only shots where both barometers have a clear arrival in the time-frequency domain are used for this computation and depicted in the figure. Since there are uncertainties in the positions of the barometers and the angle of the tether connecting them, we deduce a range of potential lag times to include the top $10 \%$ of the cross-correlation values, 
which yield a range in arrival angles, and are denoted by the error bars in Fig. 4. The arrival angles deduced from the data show the same trend as the elevation angles deduced from GPS. The error bars on the arrival angles are notably large - this is an effect of using the spectrogram to perform the time correlation as opposed to the time-domain signal itself, where the signal is too noisy to perform a reliable correlation. The spectral correlation produces a wide peak over a number of scales, resulting in a large range in angles. Shots that are more clearly detected by the barometer produce narrower correlations and more accurate angle estimations.

Once a range of angles is deduced, a cone originating from the upper barometer may be projected on the ground, which localizes a source to within an annulus. With multiple balloons, overlapping annuli may be utilized to more accurately geolocate the source. Bowman and Albert [39] have previously deployed four balloons simultaneously in an attempt to estimate the azimuth to the source of multiple controlled explosions and were able to calculate the backazimuth of one of the explosions. In our experiment, the shots were highly repeatable and the balloon was in a different position for each shot-this allowed us to treat all the shots as a single shot, with a virtual balloon at the aerostat's position during each shot. This resulted in a virtual array of balloons and annuli produced as a result of the analysis above could be overlapped with the intention of finding the seismic hammer within the region of the maximum overlap. Fig. 5 shows the result of this analysis, whereby the seismic hammer is located in the region of maximal overlap between 26 annuli corresponding to 26 shots. The peak overlap region occurs somewhat northeast of the hammer location, which is a result of the balloon flying predominantly north and east of the hammer site, thereby introducing a bias in the overlapping annuli. The figure also shows the probability of finding the seismic hammer within a $1 \mathrm{~m} \times 1 \mathrm{~m}$ on the ground. Here, the hammer is located at the southwestern end of the region with peak probability density. We can convert the overlap into a probability measure using Bayesian analysis, described in detail in Section II-E of the supplementary material. If the interpolation between known GPS positions is assumed to be perfect, the source can be localized with $90 \%$ probability within an ellipse with semimajor axis approximately $80 \mathrm{~m}$, the boundary of which is shown with a yellow dashed line in Fig. 5.

It is worth noting that the use of 26 shots is equivalent to the use of a 26 balloon array to geolocate the seismic hammer. However, several factors serve to make the geolocation process challenging - the signal strength is poor, the correlation peaks are broad, and the balloon itself does not span a very large area, therefore consecutive annuli have a significant degree of overlap and reveal less additional information about the location of the source than is ideal. Several kilometers higher in the upper troposphere and stratosphere, with balloons separated by multiple kilometers and lower background noise, an array of 3-4 balloons should theoretically be sufficient to geolocate a seismic source on the surface. On Venus, one could determine whether quakes originated in the smooth plains or proximal tectonic ridges surrounding them with the ability to geolocate them within a radius of $50 \mathrm{~km}$.

\section{Discussion AND CONCLUSION}

We have presented a detailed analysis of the very first foray into performing seismology from a balloon. In [20], we presented our initial findings and showed the detection of seismoacoustic infrasound signals on balloon-borne barometers. The current work sought to build on the results in [20] by investigating the ability of balloon-based barometers to act as proxies for surface-deployed seismometers and geophones. Our investigation into the use of airborne barometers as flying geophones has yielded promising results- the acoustic signals recorded from artificially generated seismic events reflect much of the infrasonic spectral character of the ground motion that generated them. Furthermore, the signals arrived at the time expected based on the position of the barometers relative to the source. Using two barometers on a tether, we were able to distinguish waves propagating upward from the ground; using the propagation direction, spectral content, and timing, we were able to discriminate surface-generated waves from background noise. Furthermore, using the repeatability of the shots, we were able to generate an artificial aperture to localize the seismic source. To the best of authors' knowledge, the capability to geolocate and spectrally characterize seismic activity has never been demonstrated for a balloon-based sensor platform before.

The primary goal of our campaign is to demonstrate the detection of natural earthquakes from the earth's stratosphere in order to establish the feasibility of infrasonic remote sensing for seismic activity from a balloon platform. In order to achieve this goal, we are proceeding in an incremental fashion, steadily increasing the strength of our seismic source (energies ranging from $\sim 0.1 \mathrm{MJ}$ to $\sim 100 \mathrm{GJ}$ ) and the distance at which the barometers are placed (ranging from $\sim 300 \mathrm{~m}$ to $\sim 50 \mathrm{~km}$ ). We will soon deploy balloon-based infrasound sensors over subsurface chemical explosions in order to detect seismoacoustic infrasound waves produced by them. Furthermore, we will conduct a campaign of stratospheric overflights of seismically active regions in the United States to detect naturally occurring seismic infrasound. With these stronger sources, we also hope to discriminate different seismic phases from each other, which will offer greater insight into the morphology of the subsurface.

This experiment serves as a proof of concept that balloonborne barometers can detect and study ground motion from its far-field pressure signature. However, there are nontrivial challenges prior to the application of this technology on Venus and other solar system bodies. Wind noise presents a formidable challenge in this endeavor, as shown in the results above. It has been observed that the wind noise on a floating balloon in the stratosphere is much lower than the troposphere [17], [18]. With 60-km altitude still within its troposphere, Venus' turbulent, superrotating atmosphere presents adverse wind conditions, some fraction of which are ameliorated by the ability of the balloon to drift with the prevailing wind. We are developing software and hardware techniques to reduce wind noise and improve detection and geolocation statistics. The propagation paths of infrasound signals over a planetary scale are not as direct as those presented in this experiment-the precise resolution of 
propagation paths requires reliable simulations with accurate knowledge of the planet's atmosphere. Infrasound produced by other naturally occurring phenomena has previously been detected by ground-based stations on earth from a number of originating events such as earthquakes [28], [40], [41], volcanic eruptions [42]-[45], thunderstorms [46], and meteoroids [47] — distinguishing these source mechanisms from each other is also a challenge that is being actively addressed by building a repository of signals from these events measured from balloons. To this end, the authors are integrating additional sensors into their infrasound package and are conducting multiple flight campaigns with infrasound barometers as standalone and secondary payloads on balloons around the world. The eventual aim for this technology is to be integrated on a Venus or Titan balloon mission to map shallow quakes and seismically active regions on planetary bodies. This type of mission will also benefit greatly from synergistic operation with an orbiter, which can provide planetary-scale context to local observations. One possible orbiter that performs seismology with a global context is the Venus Airglow Measurements and Orbiter for Seismicity (VAMOS) concept [48], [49]. Our initial results show tremendous promise-further development of this novel technology will enable the study of the interior structure of planetary bodies without landing on them.

\section{ACKNOWLEDGMENT}

The authors would like to thank the reviewers of this paper for their insightful comments. They would also like to thank G. Walsh, G. Savastano, S. Kedar, K. Barrow, S. Bandyopadhyay, and others from Jet Propulsion Laboratory (JPL), NASA Armstrong Flight Research Center, and HH Seismic for their support leading up to and during the Pahrump experiment. They would also like to thank the Front Sight Firearms Training Institute for providing us with the test site for this experiment. The views expressed in this paper do not necessarily represent the views of the U.S. Department of Energy or the United States Government. Barometer data used to generate results presented are available on a publicly accessible FigShare repository with doi:10.6084/m9.figshare.6137507. Seismometer data used are available at a FigShare repository with doi: $10.6084 / \mathrm{m} 9$. figshare.9118490.

\section{REFERENCES}

[1] R. Lopes and T. K. P. Gregg, Volcanic Worlds: Exploring The Solar System's Volcanoes (Springer Praxis Books). Berlin, Germany: Springer, 2004.

[2] A. T. Wood, R. B. Wattson, and J. B. Pollack, "Venus: Estimates of the surface temperature and pressure from radio and radar measurements," Science, vol. 162, no. 3849, pp. 114-116, 1968.

[3] T. Navarro, G. Schubert, and S. Lebonnois, "Atmospheric mountain wave generation on Venus and its influence on the solid planet's rotation rate," Nature Geosci., vol. 11, pp. 487-491, Jul. 2018.

[4] J. H. Woodhouse and A. M. Dziewonski, "Mapping the upper mantle: Three-dimensional modeling of Earth structure by inversion of seismic waveforms," J. Geophys. Res., Solid Earth, vol. 89, no. B7, pp. 5953-5986, 1984.

[5] A. M. Dziewonski and J. H. Woodhouse, "Global images of the Earth's interior," Science, vol. 236, no. 4797, pp. 37-48, 1987.

[6] B. Romanowicz, "Using seismic waves to image Earth's internal structure," Nature, vol. 451, pp. 266-268, Jan. 2008.
[7] D. Borisov, R. Modrak, F. Gao, and J. Tromp, "3D elastic full-waveform inversion of surface waves in the presence of irregular topography using an envelope-based misfit function," Geophysics, vol. 83, no. 1, pp. R1-R11, 2018.

[8] W. B. Banerdt et al., "InSight: A discovery mission to explore the interior of Mars," in Proc. Lunar Planetary Sci. Conf., vol. 44, Mar. 2013, p. 1915.

[9] P. G. Neudeck, R. D. Meredith, L. Chen, D. J. Spry, L. M. Nakley, and G. W. Hunter, "Prolonged silicon carbide integrated circuit operation in Venus surface atmospheric conditions," AIP $A d v$., vol. 6, no. 12, Dec. 2016, Art. no. 125119.

[10] D. Lukco et al., "Chemical analysis of materials exposed to Venus temperature and surface atmosphere," Earth Space Sci., vol. 5, no. 7, pp. 270-284, 2018.

[11] D. J. Stevenson et al., "Probing the interior structure of Venus," Keck Inst. Space Stud., Pasadena, CA, USA, Tech. Rep., 2015.

[12] L. W. Esposito, R. G. Knollenberg, M. Y. Marov, O. B. Toon, and R. P. Turco, "The clouds are hazes of Venus," in Venus. Tucson, AZ, USA: Univ. Arizona Press, 1983, pp. 484-564.

[13] V. M. Linkin et al., "Thermal structure of the Venus atmosphere in the middle cloud layer," Science, vol. 231, no. 4744, pp. 1420-1422, 1986.

[14] R. Garcia, P. Lognonné, and X. Bonnin, "Detecting atmospheric perturbations produced by Venus quakes," Geophys. Res. Lett., vol. 32, no. 16, 2005, Art. no. L16205.

[15] J. W. Coffman, "A balloon-borne microphone system," in Proc. AFCRL Sci. Balloon Symp., 1965, pp. 274-427.

[16] J. W. Wescott, "Acoustic detection of high-altitude turbulence," in Proc. AFCRL Sci. Balloon Symp., 1965, pp. 1-62.

[17] D. C. Bowman and J. M. Lees, "Infrasound in the middle stratosphere measured with a free-flying acoustic array," Geophys. Res. Lett., vol. 42, no. 22, pp. 10010-10017, 2015.

[18] D. C. Bowman and J. M. Lees, "A comparison of the ocean microbarom recorded on the ground and in the stratosphere," J. Geophys. Res., Atmos., vol. 122, no. 18, pp. 9773-9782, 2017.

[19] D. Bowman et al., "Geoacoustic observations on drifting balloon-borne sensors," in Infrasound Monitoring for Atmospheric Studies. Cham, Switzerland: Springer, 2019, pp. 125-171.

[20] S. Krishnamoorthy et al., "Detection of artificially generated seismic signals using balloon-borne infrasound sensors," Geophys. Res. Lett., vol. 45, no. 8, pp. 3393-3403, 2018.

[21] J. B. Hampshire and J. O'Donnell, "The seismic hammer," in Proc. Seismic Instrum. Technol. Symp., Albuquerque, NM, USA, 2013.

[22] H. Kanamori, "The energy release in great earthquakes," J. Geophys. Res., vol. 82, no. 20, pp. 2981-2987, 1977.

[23] W. Menke, "Applications of the POCS inversion method to interpolating topography and other geophysical fields," Geophys. Res. Lett., vol. 18 , no. 3, pp. 435-438, 1991.

[24] J. Zhang et al., "Restoration of clipped seismic waveforms using projection onto convex sets method," Sci. Rep., vol. 6, Dec. 2016, Art. no. 39056.

[25] I. Daubechies, Ten Lectures on Wavelets. Philadelphia, PA, USA: SIAM, 1992.

[26] T. Christopher and G. P. Compo, "A practical guide to wavelet analysis," Bull. Amer. Meteorol. Soc., vol. 79, no. 1, pp. 61-78, 1998.

[27] D. T. Blackstock, Fundamentals of Physical Acoustics. Hoboken, NJ, USA: Wiley, 2000.

[28] S. J. Arrowsmith et al., "A seismoacoustic study of the 2011 January 3 Circleville earthquake," Geophys. J. Int., vol. 189, no. 2, pp. 1148-1158, 2012.

[29] K. R. Jones, R. W. Whitaker, and S. J. Arrowsmith, "Modelling infrasound signal generation from two underground explosions at the source physics experiment using the Rayleigh integral," Geophys. J. Int., vol. 200, no. 2, pp. 779-790, 2015.

[30] J. Gilles, "Empirical wavelet transform," IEEE Trans. Signal Process., vol. 61, no. 16, pp. 3999-4010, Aug. 2013.

[31] J. R. Banister and W. V. Hereford, "Observed high-altitude pressure waves from an underground and a surface explosion," J. Geophys. Res., Atmos., vol. 96, no. D3, pp. 5185-5193, 1991.

[32] L. Martire et al., "Numerical simulation of the atmospheric signature of artificial and natural seismic events," Geophys. Res. Lett., vol. 45, no. 21, pp. 12085-12093, 2018.

[33] C. Torrence and P. J. Webster, "Interdecadal changes in the ENSOmonsoon system," J. Climate, vol. 12, no. 8, pp. 2679-2690, 1999.

[34] A. Grinsted, J. C. Moore, and S. Jevrejeva, "Application of the cross wavelet transform and wavelet coherence to geophysical time series," Nonlinear Processes Geophys., vol. 11, nos. 5-6, pp. 561-566, 2004. 
[35] S. Bancroft, "An algebraic solution of the GPS equations," IEEE Trans. Aerosp. Electron. Syst., vol. AES-21, no. 1, pp. 56-59, Jan. 1985.

[36] J. L. Awange and E. W. Grafarend, "Algebraic solution of GPS pseudoranging equations," GPS Solutions, vol. 5, pp. 20-32, Apr. 2002.

[37] T. Roos, P. Myllymäki, H. Tirri, P. Misikangas, and J. Sievänen, "A probabilistic approach to WLAN user location estimation," Int. J. Wireless Inf. Netw., vol. 9, pp. 155-164, Jul. 2002.

[38] D. R. Christie and P. Campus, "The IMS infrasound network: Design and establishment of infrasound stations," in Infrasound Monitoring for Atmospheric Studies. Dordrecht, The Netherlands: Springer, 2009, pp. 29-75.

[39] D. C. Bowman and S. A. Albert, "Acoustic event location and background noise characterization on a free flying infrasound sensor network in the stratosphere," Geophys. J. Int., vol. 213, no. 3, pp. 1524-1535, 2018.

[40] J. M. Young and G. E. Greene, "Anomalous infrasound generated by the Alaskan earthquake of 28 March 1964," J. Acoust. Soc. Amer, vol. 71, no. 2, pp. 334-339, 1982.

[41] J. P. Mutschlecner and R. W. Whitaker, "Infrasound from earthquakes," J. Geophys. Res., Atmos., vol. 110, no. D1, 2005, Art. no. D01108.

[42] J. B. Johnson and M. Ripepe, "Volcano infrasound: A review," J. Volcanology Geotherm. Res., vol. 206, no. 3, pp. 61-69, 2011.

[43] R. S. Matoza et al., "Seismo-acoustic wavefield of strombolian explosions at Yasur volcano, Vanuatu, using a broadband seismo-acoustic network, infrasound arrays, and infrasonic sensors on tethered balloons," J. Acoust. Soc. Amer., vol. 141, no. 5, p. 3566, 2017.

[44] J. F. Anderson, J. B. Johnson, A. L. Steele, M. C. Ruiz, and B. D. Brand, "Diverse eruptive activity revealed by acoustic and electromagnetic observations of the 14 July 2013 intense vulcanian eruption of Tungurahua Volcano, Ecuador," Geophys. Res. Lett., vol. 45, no. 7, pp. 2976-2985, 2018.

[45] R. Matoza, D. Fee, D. Green, and P. Mialle, "Volcano infrasound and the international monitoring system," Infrasound Monitoring for Atmospheric Studies. Cham, Switzerland: Springer, 2019, pp. 1023-1077.

[46] O. D. Lamb, J. M. Lees, and D. C. Bowman, "Detecting lightning infrasound using a high-altitude balloon," Geophys. Res. Lett., vol. 45, no. 14, pp. 7176-7183, 2018.

[47] W. N. Edwards, "Meteor generated infrasound: Theory and observation," in Infrasound Monitoring for Atmospheric Studies. Dordrecht, The Netherlands: Springer, 2009, pp. 361-414.

[48] A. Didion et al., "Remote sensing of Venusian seismic activity with a small spacecraft, the VAMOS mission concept," in Proc. IEEE Aerosp. Conf., Mar. 2018, pp. 1-14.

[49] B. M. Sutin et al., "VAMOS: A smallsat mission concept for remote sensing of Venusian seismic activity from orbit," Proc. SPIE, vol. 10698, Jul. 2018, Art. no. 106985T.

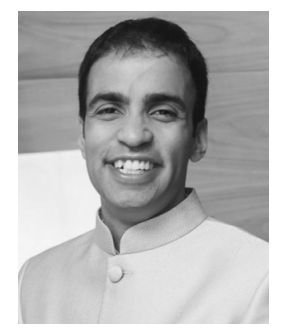

Siddharth Krishnamoorthy received the master's degree in physics from IIT Delhi, New Delhi, India in 2007, and the master's and Ph.D. degrees in aeronautics and astronautics from Stanford University, Stanford, CA, USA, in 2012 and 2017, respectively.

$\mathrm{He}$ is with the Ionospheric and Atmospheric Remote Sensing Group, NASA Jet Propulsion Laboratory, Pasadena, CA, USA, where he is developing remote sensing technologies for Venus. Since July 2017, he has been a Post-Doctoral Associate with the NASA's Jet Propulsion Laboratory (JPL), California Institute of Technology, Pasadena, CA, USA. He has a diverse background in building, testing, and deploying software and hardware solutions to study complex multiphysics problems. His research interests include instrumentation, novel remote sensing techniques, and developing enabling technologies for planetary exploration.

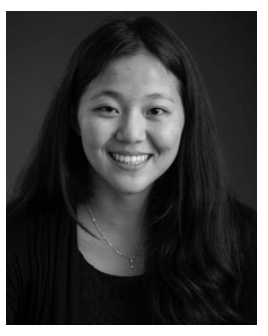

Voon Hui Lai received the B.A. degree in geophysics from the University of California at Berkeley, Berkeley, CA, USA, in 2014, and the M.Sc. degree in geophysics from the California Institute of Technology (Caltech), Pasadena, CA, USA, in 2016, where she is currently pursuing the $\mathrm{Ph} . \mathrm{D}$. degree in geophysics.

Her research interests include seismic imaging of earth structure and source analysis of unconventional events including debris flow, induced seismicity, and volcanic eruptions.

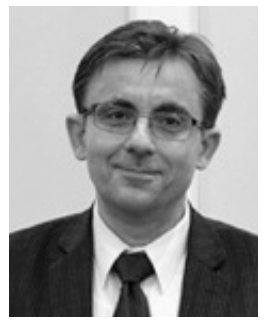

Attila Komjathy received the Ph.D. degree from the Department of Geodesy and Geomatics Engineering, University of New Brunswick, Fredericton, NB, Canada, in 1997.

He was a Post-Doctoral Researcher with the University of Colorado at Boulder, Boulder, CO, USA. $\mathrm{He}$ serves as a Group Leader and the Principal Investigator with the Ionospheric and Atmospheric Remote Sensing Group of the Tracking Systems and Applications Section, NASA's Jet Propulsion Laboratory (JPL), California Institute of Technology, Pasadena, CA, USA. He was with JPL, where he is specializing in various aspects of terrestrial and planetary remote sensing techniques over 18 years. He was an Adjunct Professor with the University of New Brunswick.

Dr. Komjathy is a fellow of the U.S. Institute of Navigation. He was a recipient of several NASA awards including the Exceptional Space Act Award, the Best Paper Awards, and the JPL Group Achievement Awards. He serves as an Editorial Board Member for the Japanese Journal Earth, Planets and Space and as an Associate Editor for Radio Science, a publication of the American Geophysical Union.

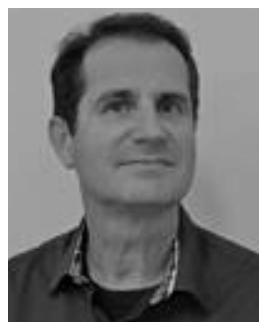

Michael T. Pauken received the B.S. degree from Vanderbilt University, Nashville, TN, USA, in 1983 and the M.S. and Ph.D. degrees in mechanical engineering from the Georgia Institute of Technology, Atlanta, GA, USA, in 1990 and 1994, respectively.

$\mathrm{He}$ is currently a Senior Technologist with the Mechanical Systems Engineering Division, Jet Propulsion Laboratory (JPL), Pasadena, CA, USA. His research interests include the development of planetary aerial vehicle systems for exploring Venus, Mars and Titan from within their respective atmospheres.

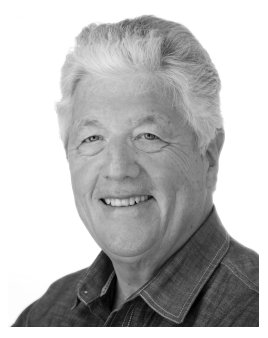

James A. Cutts received the B.A. degree in physics from Cambridge University, Cambridge, U.K. in 1965, the M.S. degree in geophysics and the Ph.D. degree in planetary science from the California Institute of Technology (Caltech), Pasadena, CA, USA, in 1967 and 1971, respectively. He received a Certificate from UCLA Executive Management Program.

He was the Manager with the Planetary Science Institute, Science Applications International Corporation, and a Scientific Investigator with the mariner 9 and Viking orbiter imaging teams. From 1988 to 1990, he has served as the Chair for the NASA Sensor Working Group, Pasadena, CA, USA, and Tucson, AZ, USA. He has also served on other NASA and U.S. Air Force advisory committees. From 1988 to 1991, he was the Program Manager of advanced concepts and the Deputy Director of the Center for Space Microelectronics Technology, Jet Propulsion Laboratory, Pasadena, CA, USA. He is currently the Program Manager with the Solar System Exploration Directorate, NASA's Jet Propulsion Laboratory (JPL), California Institute of Technology, Pasadena, CA, USA, where he is responsible for the development and demonstration of advanced concepts for solar system exploration. A major focus of this effort is planetary aerobots or robotic balloons. He has authored approximately 50 papers in planetary science, sensor technology, and innovative space mission concepts.

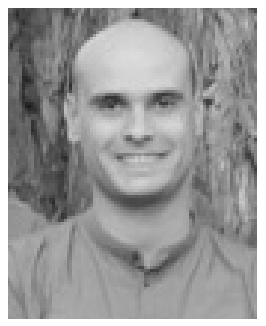

Raphaël F. Garcia is currently an Academic Researcher with the Institut Supérieur de l'Aéronautique et de l'Espace-SUPAERO, Toulouse, France. He is also a Seismologist on duty for NASA's InSight mission and is interested in studying planetary interiors and surface-atmosphere coupling on planetary bodies. 


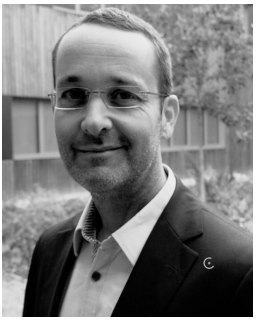

David Mimoun was a Guest Researcher with the NASA Jet Propulsion Laboratory, California Institute of Technology, Pasadena, CA, USA, from 2013 to 2014. He is currently an Associate Professor of space systems and planetary science with the Institut Supérieur de l'Aéronautique et de l'Espace (ISAE)-SUPAERO, Toulouse, France. He is also the PI of the first CubeSat of the ISAE-SUPAERO, EntrySat. $\mathrm{He}$ is also the Co-Investigator of the InSight mission, focusing on overall mission performance. He is also a Collaborator of NASA's upcoming Martian Rover (Mars 2020), with the supply of the very first Martian microphone on board SuperCam. His research interests include the design of space mission and space instruments for the geophysical exploration of the solar system.

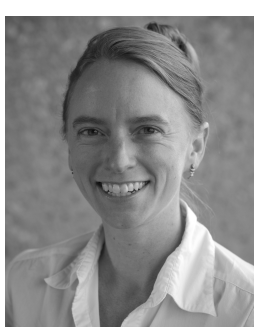

Jennifer M. Jackson received the Ph.D. degree in geology from the University of Illinois at UrbanaChampaign, Champaign, IL, USA, in 2005.

She is currently a Professor of mineral physics with the Seismological Laboratory, California Institute of Technology, Pasadena, CA, USA. Her research interests include combining experimental results with geophysical methods to better understand planetary interiors.

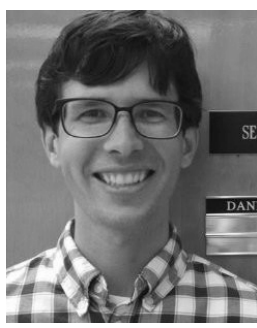

Daniel C. Bowman received the Ph.D. degree in geophysics from the University of North Carolina at Chapel Hill, Chapel Hill, NC, USA, in 2016, and the B.A. degree in geology from the Macalester College, Saint Paul, MN, USA, in 2008.

$\mathrm{He}$ is currently a Geophysicist and Atmospheric Scientist with the Sandia National Laboratories, Albuquerque, NM, USA. His research interests include atmospheric science, infrasound generation and propagation, and balloon technology development for high-altitude infrasound measurments.

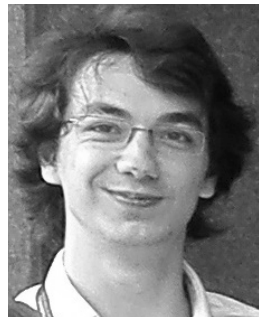

Ervan Kassarian is currently pursuing the master's degree in aerospace engineering with the Institut Supérieur de l'Aéronautique et de l'EspaceSUPAERO, Toulouse, France.

Fascinated by space exploration, he interned in the Jet Propulsion Laboratory (JPL), Pasadena, CA, USA, for 6 months in the Atmospheric and Ionospheric Remote Sensing Division. His research interests include the dynamics and control of space systems.

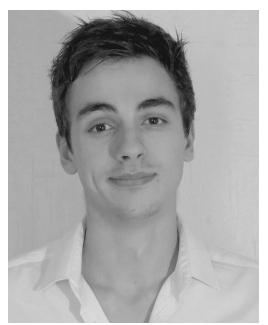

Léo Martire received the M.Sc. degree in engineering (applied mathematics) from the Institut National des Sciences Appliquées (INSA), Toulouse, France, and the M.Sc. degree in optimization from the Université Paul Sabatier, Toulouse, in 2017. He is currently pursuing the Ph.D. degree with the Institut Supérieur de l'Aéronautique et de l'EspaceSUPAERO, Toulouse, with a focus on the coupling between the ground and atmosphere systems.

$\mathrm{He}$ is currently a Science Team Member for NASA's InSight mission to Mars, where he is involved in investigating Venus exploration and earth-based studies via balloon platforms. His research interests include computational fluid dynamics simulations at geophysical scales, particularly in planetary atmospheric science and infrasound generation and propagation.

Anthony Sournac is currently a Research Engineer with the Institut Supérieur de l'Aéronautique et de l'Espace-SUPAERO, Toulouse, France.

Alexandre Cadu is currently a Research Engineer with the Institut Supérieur de l'Aéronautique et de l'Espace-SUPAERO, Toulouse, France. 